\title{
Central peak and acoustic anomalies of the relaxor ferroelectric lead magnesium niobate-lead titanate single crystal studied by the micro- Brillouin scattering
}

\author{
Ghulam Shabbir ${ }^{a)}$ \\ Physics Division, Directorate of Science, PINSTECH, Islamabad 45650, Pakistan \\ Seiji Kojima \\ Ultra-Broadband Spectroscopy Laboratory, Institute of Materials Science, University of Tsukuba, Tsukuba, \\ Ibaraki 305-8573, Japan
}

(Received 7 June 2007; accepted 11 July 2007; published online 8 August 2007)

\begin{abstract}
Quasielastic central peak and acoustic anomalies in a [001] lead magnesium niobate-lead titanate single crystal have been investigated by the high-resolution micro-Brillouin scattering. Two acoustic anomalies were observed at temperatures, $T_{C-T} \sim 415 \mathrm{~K}$ and $T_{T-R} \sim 348 \mathrm{~K}$. The damping showed a significant dispersion at $T \sim 550 \mathrm{~K}$, associated with the enhanced dynamics of local strain fields created by the polar microregions. The temperature evolution of the quasielastic central peak exhibited anomalies in the overall intensity and relaxation time that were associated with the line shape interferences of the central peak and the acoustic mode. (C) 2007 American Institute of Physics. [DOI: 10.1063/1.2768306]
\end{abstract}

The complex perovskite relaxor ferroelectric (FE) system $\mathrm{Pb}\left(\mathrm{Mg}_{1 / 3} \mathrm{Nb}_{2 / 3}\right) \mathrm{O}_{3}(\mathrm{PMN})$ and its solid solutions with $\mathrm{PbTiO}_{3}$ (PT) such as, $(1-x) \mathrm{Pb}\left(\mathrm{Mg}_{1 / 3} \mathrm{Nb}_{2 / 3}\right) \mathrm{O}_{3}-x \mathrm{PbTiO}_{3}$ $(\mathrm{PMN}-x \mathrm{PT}) \quad$ and $\quad(1-x) \mathrm{Pb}\left(\mathrm{Zn}_{1 / 3} \mathrm{Nb}_{2 / 3}\right) \mathrm{O}_{3}-x \mathrm{PbTiO}_{3}$ $(\mathrm{PZN}-x \mathrm{PT})$ have been a subject of extensive investigations owing to both their exceptionally strong piezoelectric properties and quest for a comprehensive understanding of the nature of relaxor FE materials. ${ }^{1,2}$ In the phase diagram, compositions of the most practical interest lie in the low symmetry monoclinic region called the morphotophic phase boundary (MPB); separating rhombohedral $(R 3 m)$ and tetragonal $(P 4 m m)$ FE phases. Relaxor FE single crystals near this boundary exhibit unusual high piezoelectric constant, electromechanical coupling coefficient, and ultrahigh strains ${ }^{3,4}$ when poled along the pseudocubic [001] direction which is different from the polarization directinon ([111]).

The most probable scenario toward understanding these extraordinary piezoelectric properties of the relaxor FE state stems in the well established existence of unique entities the so-called polar microregions (PMRs) with randomly oriented local polarization, appearing at/above Burns temperature $T_{B}$ (Ref. 5) $(\sim 620 \mathrm{~K}$ for PMN) in a disordered (site and/or charge) matrix that give rise to local quenched random fields, and hence transition to a long range ordered state is prevented. The relaxor FE materials are also fascinating because they resemble glasses in the sense that they exhibit aging, memory, rejuvenation, ${ }^{6-9}$ and Vogel-Fulcher-type relaxation $^{10,11}$ phenomena. However, in contrast to glasses, these characteristics have been observed in relaxors not only in the frozen regime but also even above the susceptibility maximum temperature $T_{m}(\sim 265 \mathrm{~K}$ for $\mathrm{PMN})$. At the same time the relaxor state can be switched to FE long range ordered state by applying sufficiently high electric fields, adding PT (a classic soft mode FE) to varing concentrations, and exhibiting the presence of some criticality. This has lead to

\footnotetext{
a) Author to whom correspondence should be addressed; electronic mail: gshabbir@pinstech.org.pk and gshabbir@gmail.com
}

recent findings that the presence of critical behavior in the electric field-temperature-composition phase diagram of PMN- $x$ PT (and possibly in other relaxors) is at the heart of this giant electromechanical response near the MPB. ${ }^{12} \mathrm{Cou}-$ pling between the optical and acoustic phonons in PMN (Ref. 13) and PMRs and TA phonons in PZN- $x$ PT (Ref. 14) has been associated with the dynamics of ion off-centering and appearance of PMRs, respectively. In the Raman study of the PZN crystal, both relaxation and coupled phonon dynamics have been identified. ${ }^{15}$ Recent light scattering studies on PZN- $x$ PT crystal have indicated the existence of an intermediate temperature at which the coupling between the PMRs and acoustic modes becomes more visible. ${ }^{16}$ In this letter we present a direct evidence of mode coupling that gives rise to line shape anomalies in the light scattering spectra of the PMN-33\%PT [001]-oriented single crystal using the high-resolution micro-Brillouin scattering.

PMN-33\%PT single crystal, grown by the Bridgman method, was put inside a cryostat cell (THMS 600) placed on the stage of an optical microscope (Olympus BH-2). The phonon modes were excited by a single frequency $\mathrm{Ar}^{+}$-ion laser at a wavelength of $514.5 \mathrm{~nm}$ and a power of $\sim 100 \mathrm{~mW}$. The micro-Brillouin light scattering experiments were performed in the backward scattering geometry by using a six pass tandem Fabry-Pérot interferometer from room temperature to $T \sim 650 \mathrm{~K}$ with a stability of $\pm 0.1 \mathrm{~K}$ and an accuracy of $\pm 1 \mathrm{~K}$. For accurate detection of the acoustic anomaly the Brillouin spectra were recorded by employing a smaller free spectral range (FSR) of $30 \mathrm{GHz}$ while a relatively larger FSR of the order of $100 \mathrm{GHz}$ was used to observe the quasielastic central peak (CP).

Figure 1 shows the typical Brillouin spectra of PMN$33 \%$ PT [001] single crystal at some selected temperatures. These spectra were measured in the scattering geometry, $c(a, a+b) \bar{c}$, where $a, b$, and $c$ represent [100], [010], and [001] directions, respectively. One longitudinal acoustic (LA) mode and an additional transverse acoustic (TA) mode coupled with the quasielastic $\mathrm{CP}$ were observed in this geometry. The Brillouin frequency shift $\left(\nu_{\mathrm{LA}}\right)$ and the hyper- 


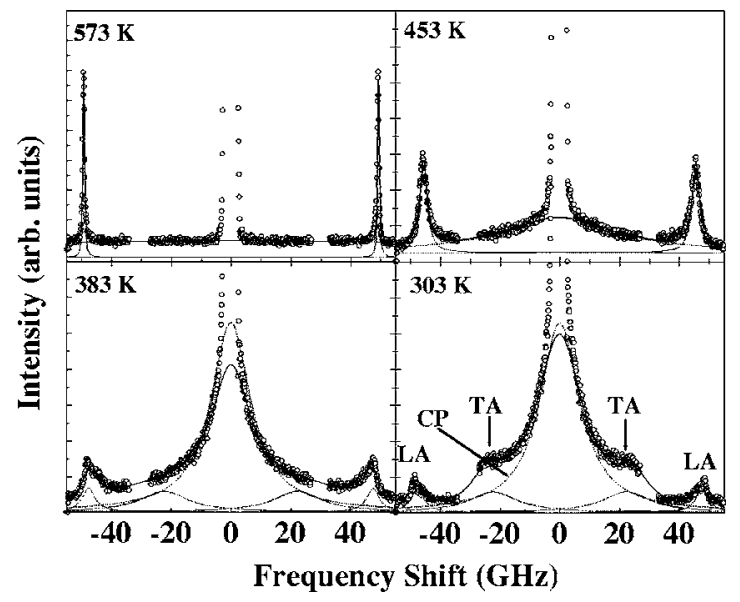

FIG. 1. Brillouin spectra of a PMN-33\%PT single crystal at some selected temperatures. The circles show the experimental data and solid line between the circles is the fitting result.

sonic damping $\left(\Gamma_{\mathrm{LA}}\right)$ of the LA mode obtained from these spectra have been plotted in Fig. 2 as a function of temperature. The acoustic velocities of LA and TA modes are controlled by the respective elastic constants, e.g., $c_{11}$ and $c_{44}$, in the cubic phase. The measured values of $c_{11}$ and $c_{44}$ for $T$ $\geqslant T_{C-T}$, say, at $\sim 453 \mathrm{~K}$, were 163 and $77 \mathrm{GPa}$, respectively. The existence of different acoustic anomalies can be clearly differentiated from these data. It is also important to mention that by analyzing the spectra presented in Fig. 1 with the damped harmonic oscillator (DHO) couple mode scheme (described below) or uncoupled DHO or Voigt function reproduced similar results. The reason lies in the fact that LA mode is hardly affected by the $\mathrm{CP}$ and/or the TA mode.

It can be observed from Fig. 2 that with decreasing temperature, $\Gamma$ deviates from linearity and begins to increase at $T \leqslant 550 \mathrm{~K}$, whereas $\nu_{\mathrm{LA}}$ shows softening comparatively at a much higher temperature (beyond the measured temperature). It is remarkable to note that this dispersion in $\Gamma$ and $\nu_{\mathrm{LA}}$ exhibits similar temperature dependencies to that of PLZT relaxor FE ceramics. ${ }^{10}$ The two subsequent acoustic anomalies occurring at temperatures $T_{C-T} \sim 415 \mathrm{~K}$ and $T_{T-R}$ $\sim 348 \mathrm{~K}$ show the paraelectric (PE) cubic-tetragonal and then tetragonal-rhombohedral FE phase transformations, respectively. This depicts that the structural changes have sig-

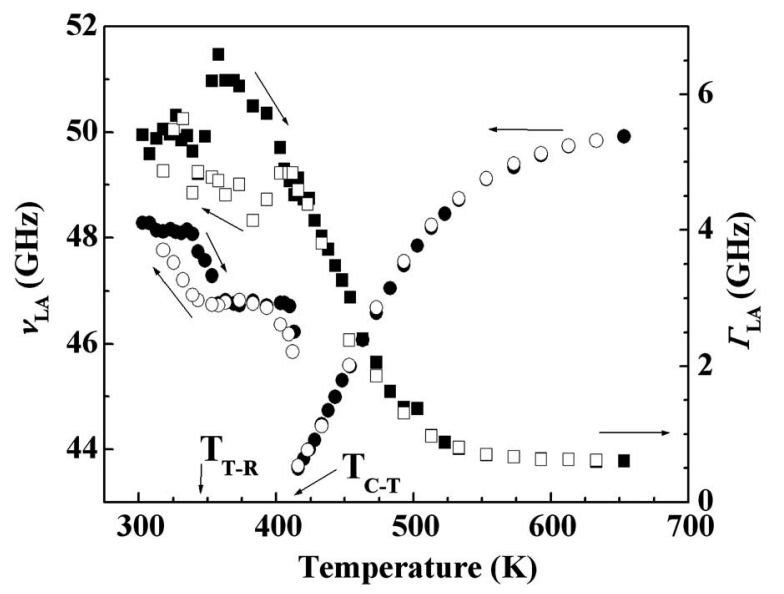

FIG. 2. Temperature dependencies of the Brillouin frequency shift $\left(\nu_{\mathrm{LA}}\right)$ (circles) and damping factor $(\Gamma)$ (squares) of the longitudinal acoustic mode

relaxation time of CP increases approaching a sharp maxi-
Downloaded 30 Nov 2009 to 130.158 .56 .186$. Redistribution subject to AlP license or copyright; see http://apl.aip.org/apl/copyright.jsp

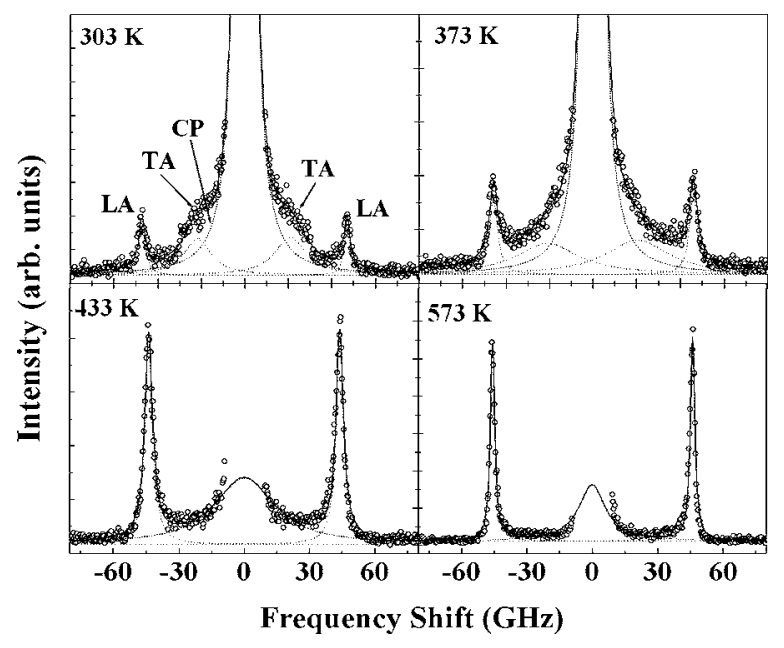

FIG. 3. Brillouin spectra of PMN-33\%PT crystal measured with a FSR of $100 \mathrm{GHz}$ in the rhombohedral $(303 \mathrm{~K})$, tetragonal $(373 \mathrm{~K})$, cubic $(433 \mathrm{~K})$, and cubic $(573 \mathrm{~K})$ with no $\mathrm{CP}$, regions, respectively.

nificant influence on the elastic behavior of the crystal. The gradual softening of $\nu_{\mathrm{LA}}$ and rise in $\Gamma$ can be attributed to the appearance (and subsequent growth) of the PMRs at/above the Burns temperature $T_{B}$, far from $T_{m}$, where the optical index of refraction deviates from the high temperature linear behavior. $^{5}$

The temperature dependent Brillouin spectra of the $\mathrm{CP}$ in the temperature regimes of interest are shown in Fig. 3. From these spectra two temperature regions can be identified: (i) $T \leqslant T_{C-T}$ and (ii) $T \geqslant T_{C-T}$, where the spectra consists of LA mode and TA mode coupled with the CP, and LA mode and the $\mathrm{CP}$, respectively. Accordingly, we have analyzed the spectra for $T \leqslant T_{C-T}$ by a damped harmonic oscillator (describing LA mode, $\left.\omega_{\mathrm{LA}}=2 \pi \nu_{\mathrm{LA}}\right)$,

$$
\chi_{1}(\omega, T) \propto\left[\omega_{\mathrm{LA}}^{2}(T)-\omega^{2}-i \omega \Gamma_{\mathrm{LA}}(T)\right]^{-1},
$$

and a DHO (describing TA mode) coupled with the Debyetype $\mathrm{CP}$ by the response function with relaxing self-energies related to the imaginary part of the dynamic susceptibility, ${ }^{17}$

$$
\chi_{2}(\omega, T) \propto\left[\omega_{\mathrm{TA}}^{2}(T)-\omega^{2}-i \omega \Gamma_{\mathrm{TA}}(T)-\frac{\delta^{2}(T)}{1-i \omega \tau}\right]^{-1},
$$

where $\tau$ is the Debye relaxation time and $\delta^{2}$ is the coupling constant, i.e., the measure of interaction strength between the quasielastic $\mathrm{CP}$ and the coupled TA mode of frequency $\omega_{\mathrm{TA}}\left(=2 \pi \nu_{\mathrm{TA}}\right)$ and damping factor $\Gamma_{\mathrm{TA}}$.

The TA mode became very weak approaching $T$ $\sim 370 \mathrm{~K}$ and ultimately disappeared in the vicinity of $T_{C-T}$. Damping $\left(\Gamma_{\mathrm{TA}}\right)$ and frequency shift $\left(\omega_{\mathrm{TA}}\right)$ of the TA mode remained unchanged for $T \leqslant T_{T-R}$ and were of the order of $\sim 4.5$ and $\sim 163 \mathrm{GHz}$, respectively; however, the same could not be obtained reliably for $T \geqslant T_{T-R}$ due to complete merging of TA mode into the CP. In the PE phase $\left(T \geqslant T_{C-T}\right)$ the fitting process was carried out with an uncoupled $\mathrm{CP}$ and the LA mode. It is important to note that the repeated fitting process yielded reproducible behavior of the fitting parameters in the two temperature regimes.

Overall intensity factor and relaxation time $(\tau)$ of $\mathrm{CP}$ as obtained from the coupled/uncoupled mode analysis of the measured spectra are shown in Fig. 4. Several things can be inferred from these data. (i) With decreasing temperature, relaxation time of $\mathrm{CP}$ increases approaching a sharp maxiO AIP license or copyright; see http://apl.aip.org/apI/copyright.jsp 


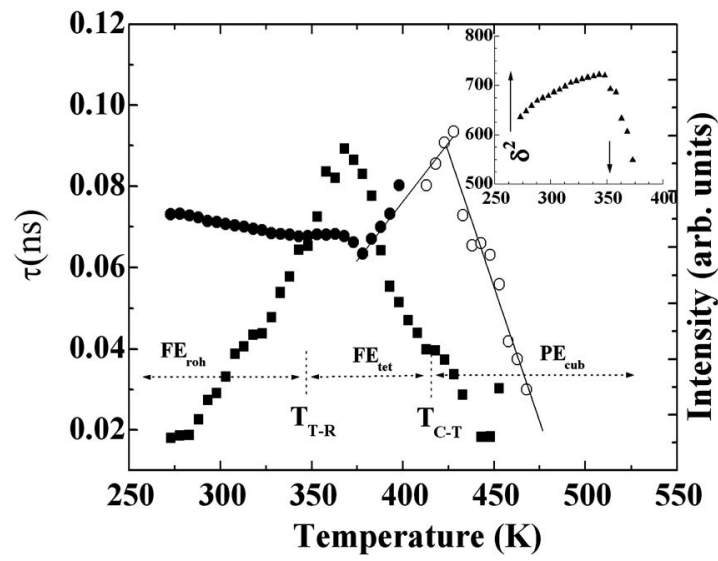

FIG. 4. Temperature dependencies of the overall intensity factor (filled squares) and relaxation time $(\tau)$ (filled circles are for coupled mode and open circles for uncoupled mode analysis) of the quasielastic central peak. Solid lines are mere guides for the eye. The inset shows the coupling constant $\delta^{2}$ as a function of temperature.

mum around $T_{C-T}$, where overall intensity factor gives a small inflection; it then decreases showing a dip at $T$ $\sim 378 \mathrm{~K}$ with a corresponding maximum in the overall intensity factor at $T \sim 370 \mathrm{~K}$ (slowing down of relaxation dynamics and growth of $\mathrm{CP}$ ). (ii) With further cooling the overall intensity factor lowers and $\tau$ shows only minor rise. (iii) The coupling constant $\delta^{2}$ is minimum, where $\tau$ has a dip $(T \sim 378 \mathrm{~K})$ and becomes highest at $T_{T-R}$, it then softens slowly with further cooling. It is also noted that the TA mode does affect the CP line shape but has no appreciable contribution to the LA mode line profile; hence the two acoustic anomalies remain unaffected by its appearance. The anomalous behavior of CP parameters (reflected in overall intensity factor and $\tau$ ) for $T \leqslant T_{C-T}$ demonstrate that it comes from the line shape interferences due to interaction of TA phonon with the quasielastic $\mathrm{CP}$. The reason behind this argument lies in the fact that when we analyzed the same data with uncoupled DHO and Debye relaxation mode, the anomalies in the $\tau$ decreased but the intensity of $\mathrm{CP}$ showed two peaks. The PMR dynamics is no more effective in this temperature range because the crystal has already been transformed to long-range ordered state. The other factor that may influence $\mathrm{CP}$ dynamics in the present case is realignment/movement of domain walls in the FE matrix resulting from the reorientation of polarization direction from [001] to [111]. ${ }^{18}$ This explanation is supported by similar remarks about PZN-8\%PT (Ref. 19) and PMN-60\%PT (Ref. 20) crystals, where the interference of acoustic and optical phonon branches gives rise to "waterfall effect" observed in PMN and PZN relaxors. Fourier transform infrared spectra ${ }^{21}$ between 20 and $900 \mathrm{~K}$ revealed the existence of an underdamped soft mode in PMN crystal. The appearance of CP below $T_{B}$ and its slowing down character on cooling down to frequencies below the microwave range has lead to the conclusion that the waterfall effect may be associated with the overlapping of the soft mode with the $\mathrm{CP}^{21}$ From the temperature dependence of the acoustic anomalies in PMN-33\% PT crystal, it can be argued that in this crystal, a certain temperature point $(T \sim 550 \mathrm{~K})$ below $T_{B}$ indeed exists where the dynamics of PMRs produce sufficient local strain fields in the crystal that result in appreciable dispersion in frequency and damping of the LA phonon mode. This observation is consistent with our previous findings on PLZT (Ref. 22) and recent light scattering studies on PZN-9\%PT crystal. ${ }^{16}$ It is about $100 \mathrm{~K}$ below this temperature where the quasielastic $\mathrm{CP}$ becomes more prominent as observed in PLZT relaxor ${ }^{23}$ and in the present case also. A similar trend of the $\mathrm{CP}$ has also been reported for PMN-32\%PT crystal by neutron scattering experiments ${ }^{24}$ and associated with the dynamical evolution of PMRs.

In summary, high-resolution micro-Brillouin scattering technique was applied to investigate the acoustic anomalies and quasielastic $\mathrm{CP}$ in a [001]-oriented PMN-33\% PT single crystal. Two acoustic anomalies, PE cubic-FE tetragonal $\left(T_{C-T} \sim 415 \mathrm{~K}\right)$ and $\mathrm{FE}$ tetragonal-FE rhombohedral $\left(T_{T-R}\right.$ $\sim 348 \mathrm{~K})$ symmetries, were observed. The LA mode data also exhibited the existence of a temperature point ( $T$ $\sim 550 \mathrm{~K}$ ), where the dynamics of PMRs becomes more prominent in PMN-33\%PT crystal and possibly in other relaxor FE materials. From the analysis of Brillouin spectra with larger FSR coupling between the acoustic mode and the quasielastic, $\mathrm{CP}$ was evident which gives rise to temperature dependent line shape anomalies in the relaxation time and overall intensity factor of the $\mathrm{CP}$. The detailed analysis of the $\mathrm{CP}$ data will be published elsewhere.

${ }^{1}$ L. E. Cross, Ferroelectrics 76, 241 (1987).

${ }^{2}$ Z.-G. Ye, Key Eng. Mater. 155-156, 81 (1998).

${ }^{3}$ S.-E. Park and T. R. Shrout, J. Appl. Phys. 82, 1804 (1997).

${ }^{4}$ J. Kuwata, K. Uchino, and S. Nomura, Jpn. J. Appl. Phys., Part 1 21, 1298 (1982).

${ }^{5}$ G. Burns and F. H. Docal, Phys. Rev. B 28, 2527 (1983).

${ }^{6}$ G. Shabbir, J.-H. Ko, and S. Kojima, Appl. Phys. Lett. 86, 012908 (2005).

${ }^{7}$ G. Shabbir and S. Kojima, J. Korean Phys. Soc. 46, 128 (2005).

${ }^{8}$ E. V. Colla, L. K. Chao, and M. B. Weissman, Phys. Rev. B 63, 134107 (2001).

${ }^{9}$ F. Cordero, F. Craciun, A. Franco, D. Piazza, and C. Galassi, Phys. Rev. Lett. 93, 097601 (2004).

${ }^{10}$ G. Shabbir and S. Kojima, Europhys. Lett. 63, 388 (2003).

${ }^{11}$ A. E. Glazounov and A. K. Tagantsev, Appl. Phys. Lett. 73, 856 (1998).

${ }^{12}$ Z. Kutnjak, J. Petzelt, and R. Blinc, Nature (London) 441, 956 (2006).

${ }^{13}$ O. Svitelskiy, J. Toulouse, and Z.-G. Ye, Phys. Rev. B 68, 104107 (2003).

${ }^{14} \mathrm{D}$. La-Orauttapong, J. Toulouse, Z.-G. Ye, W. Chen, R. Erwin, and J. L. Robertson, Phys. Rev. B 67, 134110 (2003).

${ }^{15}$ J. Toulouse, F. Jiang, O. Svitelskiy, W. Chen, and Z.-G. Ye, Phys. Rev. B 72, 184106 (2005).

${ }^{16}$ J.-H. Ko, D. H. Kim, and S. Kojima, Appl. Phys. Lett. 90, 112904 (2007).

${ }^{17}$ S. M. Shapiro, J. D. Axe, G. Shirane, and A. Riste, Phys. Rev. B 6, 4332 (1972).

${ }^{18}$ G. Shabbir, S. Kojima, and C. Feng, J. Appl. Phys. 100, 064107 (2006).

${ }^{19}$ J. Hlinka, S. Kamba, J. Petzelt, J. Kulda, C. A. Randall, and S. J. Zhang, Phys. Rev. Lett. 91, 107602 (2003).

${ }^{20}$ C. Stock, D. Ellis, I. P. Swainson, G. Xu, H. Hiraka, Z. Zhong, H. Luo, X. Zhao, D. Viehland, R. J. Birgeneau, and G. Shirane, Phys. Rev. B 73, 064107 (2006).

${ }^{21}$ S. Kamba, M. Kempa, V. Bovtun, J. Petzelt, K. Brinkman, and N. Setter, J. Phys.: Condens. Matter 17, 3965 (2005).

${ }^{22}$ G. Shabbir and S. Kojima, J. Phys.: Condens. Matter 15, 7717 (2003).

${ }^{23}$ G. Shabbir and S. Kojima, Ferroelectrics 303, 167 (2004).

${ }^{24}$ S. N. Gvasaliya, B. Roessli, R. A. Cowley, S. Kojima, and S. G. Lushnikov, J. Phys.: Condens. Matter 19, 016219 (2007). 